\title{
Role of Mri in Meniscal Tearsdr
}

\author{
Jeevika Mu ${ }^{1}$,Dr L.Vinay Bhushan Reddy ${ }^{2}$ \\ ${ }^{1}$ Head Of Radiology Department, Jjmmc Davangere,India \\ ${ }^{2}$ Second Year Radiology Resident, Jjmmc Davangere,India
}

\begin{abstract}
MR imaging provides information on soft tissue damage and has been accepted widely for the evaluation of internal knee derangements. Hence the purpose of this study was to evaluate the usefulness of MRI in the early and precise diagnosis and to study the meniscal anatomy, normal variants, types and incidence of injuries in menisci by MRI. Out of 43 patients the males (84\%) are commonly affected than females (16\%). Majority of the patients fall under the age group of 15-30 years (48\%). Among meniscal injuries medial meniscal tears (46.5\%) were more common than lateral meniscus (37.2\%), Grade III tears were common in both .Among medial meniscus tear, The posterior horn (55\%) was commonest site of involvement and the predominant type of tear was Horizontal and oblique (35\%). Among lateral meniscus tear, the anterior horn (31.3\%) was commonest site of involvement and the predominant type of tear was horizontal(31.3\%).

Interpretation And Conclusion : This prospective study consisting of 43 patients was performed in the Department of Radio-Diagnosis on patients referred from Orthopaedic department, Bapuji Hospital Davangere. They were evaluated with detailed clinical history and clinical examinations

and were subsequently subjected to imaging of knee using 1.5 Tesla Philips Achieva machine using 8-channel SENSE knee coil. The

following sequences were selected as required T1W coronal, PD Fat Sat Sagittal, STIR coronal, T2W axial.

Methods :

Normal Shape and Attachments of the Medial Meniscus- The Menisci are crescentic laminae that deepen the articulation of the tibial surfaces that receive the femur. Their peripheral attached borders are thick and convex, their free borders thin and concave. Their peripheral zone is vascularized by capillary loops from the fibrous capsule and synovial membrane, while their inner regions are avascular They are wedge-shaped, semilunar, fibrocartilage structures composed of thick collagen fibers primarily arranged circumferentially, with radial fibers extending from the capsule, between the circumferential fibers.[8]They cover 50\%

of the medial and 70\% of the lateral surface of the tibial Plateau[9]Each meniscus is divided into an anterior horn, a body, and a posterior horn.

These criteria are, first, contact of intrameniscal signal with the superior or the inferior surface of a meniscus (or with both surfaces) and, second, distortion of the normal appearance of a meniscus [7].To diagnose a meniscal tear using these criteria, it is essential to understand how normal variations in the shape of the menisci and their attachments compare with the MR appearance of a meniscal tear

Mr Criteria For Diagnosing Meniscal Tears: To study the spectrum of MRI findings meniscal tears in traumatic knee. To correlate imaging findings with clinical examination findings.To identify the commonest derangement in meniscal tears
\end{abstract}

Objectives:

Keywords: (knee, meniscal anatomy, meniscal tear, MRI, normal variants)

MRI is generally considered a valuable diagnostic tool when there is a history of knee trauma. MR imaging is the best modality

for detecting meniscal tears. It is non invasive, fast can be done on an outpatient basis and is free of complications.

\section{Introduction}

Due to its anatomical configuration and because of its being the biggest joint of the human body, the knee is frequently subjected to direct trauma that can results in injuries of variable gravity[1]. MRI is a wellaccepted imaging Modality in the diagnostic workup of patients with knee complaints and has largely replaced diagnostic arthroscopy for this purpose MRI is capable of depicting soft tissue abnormalities, such as meniscal lesions[2]. When evaluating a knee MR examination we study the medial and lateral menisci first on the sagittal images and then on the coronal images because sagittal images are the Most useful in diagnosing meniscal tears Menisci should have low signal intensity on MR images because of their fibrocartilage composition, but they may have central globular or linear increased signal intensity secondary to internal mucinous degeneration [3] In addition, the menisci in asymptomatic children have a 66\% frequency of internal signal on MRI and that signal is presumed to reflect normal vascularity [4]. Another proposed cause of increased intrameniscal signal is a 
meniscal contusion after acute trauma [5]. the diagnosis of meniscal contusion for menisci with Internal signal equal to fluid on T2-weighted images in patients with a recent episode of trauma Currently MR imaging is the best modality for meniscal tears and hence this study is undertaken to determine the Role of MRI in Meniscal tears.

If high signal clearly disrupts an articular surface of the meniscus, it is a torn Meniscus.[18] Tears in the menisci result from acute trauma or repetitive trauma and progressive degeneration. Chronic repetitive trauma is common both in athletes and nonathletes with aging. Acute tears are usually due to athletic injuries, with crushing of the meniscus between the tibia and femoral condyles. Most tears extend from posterior to anterior. Chrondrocyte necrosis and increase in mucoid ground substance can lead to meniscal tears. [44 ]MRI is an established method of diagnosing meniscal tears with high sensitivity and Specificity.[19][20] In approximately $10 \%$ of cases, however, MRI findings are equivocal for the diagnosis of meniscal tear. Evaluation of the meniscus in these instances may be limited by partial volume averaging, truncation artifact, artifact From motion, meniscal degeneration, or normal anatomic structures abutting the meniscus.[21],[22],[23]

Meniscal tears : Many variants of meniscus are seen. Commonest among them is the discoid meniscus. Discoid meniscus is an anatomical variant in which the meniscus is thickened and disc-shaped, covering a greater area of the tibial plateau than the normal semilunar meniscus.[12]Although far more common on the lateral side, it is described for both lateral and medial menisci. Discoid menisci are arthroscopically classified by Watanabe et al as complete, incomplete and Wrisberg type.[13],[14]. Various MR imaging criteria have been proposed for the diagnosis. 26 The most widely-used criterion is when three or more $4 \mathrm{~mm}$ Thick consecutive sagittal images demonstrate continuity of the meniscus between the anterior and posterior horns.[15],[16 ] An abnormal, thickened, bow-tie appearance of the meniscus may also be suggestive.[17 ]Wrisberg variant of a discoid lateral meniscus.[18] This is a discoid meniscus that lacks attachments to the capsule via the normal struts or fascicles and lacks attachment to the tibia via the coronary or meniscotibial ligaments at the posterior horn of the meniscusA true meniscal flounce or fold represents a normal variant, provided there are no other associated indicators of meniscal pathology. The presence of a meniscal flounce is not associated with an increased prevalence of meniscaltears

\section{Meniscal Variants-}

The anterior horn of the medial meniscus is attached to the tibial Plateau anterior to the anterior cruciate ligament.[9].The anterior horn of the lateral meniscus has fibers of the ACL that extend into it at the anterior root attachment where it attaches to the Tibial plateau. The posterior horn of the medial meniscus attaches to the tibial plateau immediately anterior to the posterior cruciate ligament.The circumferential arrangement of the type I collagen fibers provides the meniscus with tensile strength most essential for its load bearingfunctions[10]The menisci transmit more than $50 \%$ of body weight in extension, and even more in flexion.[11]

These are vertical longitudinal tears with displaced fragment. It occurs in about $10 \%$ of meniscal tears. Few characteristic signs are seen in this type Of tear which are described below.

Absent bow tie sign: Only one body segment is seen instead of the normal two body segments present on the outermost sagittal images through the meniscus.

Fragment in notch sign: A displaced meniscal fragment should always be found, most often in the intercondylar notch. It is seen more often in lateralbucket-handle tears,[27] and it has a sensitivity of $60 \%$ to $98 \%$ and specificity of $73 \%$ to $82 \%$ for detecting bucket-handle tears[28],[29],[30],[31].

Double PCL sign: The displaced meniscal fragment can lie in front of the posterior cruciate ligament. Anterior flipped meniscus sign: The displaced fragment may flip over the anterior horn of the affected meniscus

Bucket Handle Tear:

Oblique and horizontal tear :

It affects commonly the undersurface of the posterior horn of the medial meniscus. These are mostly degenerative in nature.

- Grade 1- Meniscal signal change is globular and do not communicate with articular surface.

- Grade 2- Meniscal signal change is linear, intrasubstance and do not communicate with articular surface. • Grade 3 - Meniscal signal change that extends to the articular surface.

$\mathbf{3 a}$ - linear intrameniscal signal intensity abutting articular surface. $\mathbf{3 b}$ - irregular signal intensity abutting articular surface.

- Grade 4 - Menisci are distorted in addition to signal changes similar to grade 3

Grading of meniscal tears[24],[25],[26]: 
Types Of Meniscal Tears

- Meniscocapular Tear

- Horizontal Cleavage Tear

- Flap Tear

- Radial/ Parrot Beak Tear

- Bucket Handle Tear

- Peripheral Tear

A. Truncated triangle sign, B. Cleft sign, C. Marching cleft sign, D. Ghost meniscus sign

Meniscal flap tear :

A meniscal flap tear or displaced flap is the result of a short-segment, horizontal meniscal tear with either superior or inferior displacement of a meniscal fragment.[40] Meniscocapsular separation occurs when the meniscus detaches from the capsular attachments,[37 ]which is more common medially and usually is associated with other injuries.[38 ] Signs that have been described in meniscocapsular separation include displacement of the meniscus relative to the tibial margin, extension of the tear into the superior or inferior corner of the peripheral meniscus, and an irregular outer margin of the meniscus body on coronal images. Additional signs include increased distance between the meniscus and the MCL, or fluid between the meniscus and the MCL.[38],[39]

\section{Meniscocapsular separation :}

A root tear occurs at the tibial attachment or "root" of the meniscus, and it has been described only posteriorly. Meniscal extrusion is more pronounced and nearly four times as common with medial, as opposed to lateral meniscal root tears.[35] Lateral meniscal root tears are diagnosed when the posterior horn of the lateral meniscus does not cover the most medial aspect of the posterior lateral tibial plateau on at least one coronal image.[36]

\section{Root tear :}

The two most effective signs were the cleft and the truncated triangle signs. The use of only these two signs increased detection of radial Meniscal tears to $76 \%$. The use of all four signs increased the detection rate for radialtears to $89 \%$.[34]

Radial tears of the meniscus are significant in that radial transection of the meniscus will completely disable the load-bearing function of the meniscus and will allow the meniscus to be extruded under axial loading.[32],[33] They are defined as vertical tears perpendicular to the free edge of the meniscus, characterized by blunting of both the anterior horn-body junction and blunting and elongation of the posterior horn body segment. Because the tearplane is in the anterior third of the meniscus, the posterior horn body segment is seen as exaggerated or elongated, a finding characteristic of radial Tears

Keith W Harper et al. described show four signs for detecting radial meniscal tears.[34]

Radial or transverse tears :

Pitfalls in imaging of meniscal injuries[41]:

Truncation artifacts are seen in the meniscus on sagittal images when using $128 \times 256$ matrix and phase encoding in the superior-inferior (SI) direction. This subtle linear area of high signal intensity is commonly identified two pixels from the meniscal joint fluid interface. This defect can be easily recognized as it can be seen extending beyond the meniscal margins when images are optimally windowed. This artifact can be removed using $256 \times 256$ or $192 \times 256$ matrix and by switching the phase direction to the AP direction.

Vacuum phenomenon is seen as irregular low intensity structure extending into the joint from the meniscal margin and can simulate discoid menisci or articular abnormalities in the joint.

Magic angle phenomenon has been described with tendons and menisci when collagen fibers are oriented approximately $55^{\circ}$ to the static magnetic field. At this angle, the interactions that contribute to T2 relaxation among water protons are nulled. This can result in increased signal intensity on the upper medial portion of the posterior horn of the lateral meniscus. This is particularly common with short TE sequences.

Secondary Signs Of Meniscal Tear[42] : Useful in equivocal cases of meniscal tear.

Meniscal/parameniscal cyst: This indirect MRI sign has a reported positive predictive value of $100 \%$ for the presence of meniscal tear at arthroscopy.

\section{Joint effusion}

3. MCL edema: This sign has nearly $100 \%$ specificity for the presence of adjacent Collateral ligament edema on MRI in relation to the presence of meniscal tear at arthroscopy. The sensitivity was higher for medial meniscal tears than for lateral meniscal tears, reflecting the closer apposition of the peripheral margin of the 
medial meniscus to the medial collateral ligament than of the lateral meniscus and lateral collateral ligament.

4. Subchondral marrow edema: This is seen immediately adjacent and parallel to the articular surface. associated with medial meniscal tears in more than $60 \%$ and lateral meniscal tears in almost $90 \%$ of cases.

Findings On Physical Examination General findings include the following :

- Clicking

- Localised tenderness along the medial or lateral joint line or over the periphery of the meniscus.

- Locking of the knee in fixed flexion.

- Pseudo-locking which may be related to muscle spasm. • Limping.

- All the patients referred with knee injuries following trauma. $\bullet$ Cases of all age groups.

Exclusion criteria :

- Patient having history of claustrophobia

- Patient having history of cardiac pacemakers, metallic foreign body and cochlear implants insitu.

Methods : Imaging protocol:

Technique - Imaging will be done with 1.5 Tesla Philips Achieva Machine using 8 channel SENSE knee coil.

Positioning - Imaging is done with full extension in neutral position. A small field of view (FOV) typically in the

range $14-16 \mathrm{~cm}$

Method-A prospective study consisting of 43 patients with traumatic knee joint is undertaken to study the spectrum of

MRI findings in all consecutive cases of knee trauma referred from orthopaedic OPD of Bapuji Hospital, Davangere

in a period of 1 years from November 2015 to November 2016.

Inclusion criteria :

To examine the lateral meniscus the examiner has to return the knee to full flexion and apply a medial rotation to the tibia prior to extending the knee once again. If pain occurs in first $30^{\circ}$ of extension, indicates tear of posterior horn. Pain at $90^{\circ}$ or above indicates tear in middle part or anterior horn

The bounce test, McMurray's test and Apley's grind test can aid in the diagnosis of Meniscal injury McMurray test :

The McMurray test is used to determine the presence of a meniscal tear. While the patient is supine on the exam table the examiner grasps the knee, placing one hand over the top of the knee with thumb over one joint line and index and middle finger over the opposite joint line. The examiner begins with the knee in full flexion and then medially and laterally rotates the tibia while paying attention for an audible click. The examiner then laterally rotates the tibia and extends the knee beyond $90^{\circ}$. An audible click while performing this maneuver can indicate a torn meniscus

- Clinical tests for menisci :Out of total 43 patients with meniscal tears $20(46.5 \%)$ were isolated medial meniscus tear, $16(37.2 \%)$ were isolated lateral meniscus tear and 7 (16.3\%) involved both menisci.

Distribution of meniscal tears

\section{Results}

Data of 43 patients were analyzed in this study

Following statistical methods were applied in the present study. 1. Descriptive statistics

2. Cross tables (contingency table analysis).

a) T2W axial - TE (100 MS), TR (5400 MS), FOV (160), Slice thickness (1-3 mm).

b) PD fat sat sagittal - TE (30 MS), TR (2500 MS), FOV (155), Slice thickness (3 mm). c) STIR coronal - TE (60 MS), TR (3547 MS), FOV (150), Slice thickness $(3 \mathrm{~mm})$.

d) mFFE sagittal - TE (9.2 MS), TR (934 MS), FOV (165), Slice thickness (1-3 mm). e) T1W coronal - TE (7 MS), TR (500 - 700 MS), FOV (150) Slice thickness (1mm).

The following sequences will be selected as required.

Distribution Of Meniscal Tears Site Of Tear Medial Meniscus

Out of 20 patients with isolated medial meniscus tear, the posterior horn was commonest site of involvement occurring in

11 patients (55\%) and the predominant type of tear was horizontal and oblique that occurred in 7 patients each $(35 \%)$.

Grade III tear were the commonest seen in 12 patient (60\%) followed by grade II in 8 patients (40\%). 
Type Of Tear- Medial Meniscus

Medial Meniscus -Grades

Lateral Menisci Site

Out of 16 patients with isolated lateral meniscus tear the anterior horn was commonest site of involvement occurring in 5 patients $(31.3 \%)$ and the predominant type of tear was horizontal and was seen in 5 patients $(31.3 \%)$. Grade III tears were the commonest and were seen in 8 patients $(50 \%)$ followed by grade II in 6 patients $(37.5 \%)$. The commonest type of tear to involve the whole of meniscus both medial and lateral was horizontal tear and site was posterior horn. Grade III tear were commonest among both

Types Of Tear Lateral Menisci correlation of meniscal tear with mcmurrays test

Fig. 2: Sagittal MR Image of Superior and Inferior Popliteo meniscal Fascicles

Fig. 1: Sagittal MR Image of Anterior and Posterior Meniscofemoral Ligaments

Fig. 3: Transverse Meniscal Ligament On Axial MR Image

Fig. 4 : Coronal MR image of discoid meniscus

(B) Sagittal MR image of a normal lateral meniscus

Fig. 5 : (A) Sagittal MR image of a normal medial meniscus

Fig. 6: Wrisberg variant of discoid meniscus on sagittal MR Image - no attachment of meniscus to capsule Fig. 7: Wavy Contour of Meniscal flounce on sagittal MR Image.

Fig 9-pd sagittal image showing bucket handle tear of the lateral meniscus with displaced fragment seen in the posterior intercondylar region

Fig 8-pd sagittal image showing horizontal tear of the body and posterior horn of medial meniscus

Fig 11- 45-year-old man with complex tear of posterior horn of medial meniscus. Coronal proton densityweighted image shows extensive distortion of meniscus

Fig 10 -stir coronal image showing grade ii mcl tear with lateral sublaxation of body of medial meniscus

FIG 12-58-year-old woman with fraying and adjacent synovitis of lateral posterior root. A and B, Sagittal (A) and coronal (B) proton density-weighted images show increased signal contacting surface of root (arrows).

FIG 13-34-year-old man with longitudinal tear (arrow). Sagittal proton density- weighted image shows that tear extends to inferior surface of menisci.

FIG 14 -14-year-old-boy with full-thickness peripheral longitudinal tear of posterior horn of lateral meniscus.

A and B, Tear (arrows) is evident on proton density-weighted image (A) but is more conspicuous as fluid cleft on $T 2$ image $(B)$.

FIG 16- 17-year-old boy with bucket-handle lateral meniscal tear. Sagittal proton density-weighted image shows fragment flipped anteriorly (arrow) to abut anterior horn creating flipped meniscus sign.

FIG 15- 37-year-old woman with bucket-handle medial meniscal tear. Midline sagittal proton densityweighted image shows displaced central fragment (arrow) paralleling posterior cruciate ligament (PCL) creating double PCL sign.

\section{Conclusion}

1. Vaz CES, Camargo OP de, Santana PJ de, Valezi AC. Accuracy of Magnetic Resonance In Identifying Traumatic

2. Intraarticular knee lesions. Clinics. 2005;60(6);445-50

3. Edwin H.G. Oei, Abida Z.Ginai, M.G. Myriam Hunink. MRI for Traumatic knee injury :A Review

4. Ravi Sohal Feb 26, 2009 - what does an MRI Scan knee show?

5. Stoller DW. Magnetic Resonance Imaging in Orthopaedics and Sports Medicine. 3rd ed. Philadelphia: Lippincott Williams \& Wilkins; 2007.

6. Standring S. Gray's Anatomy. 39th ed. Philadelphia: Elsevier Ltd; 2005. p.1475-8.

7. Anderson AF, Dome DC, Gautam S, Awh MH, Rennirt GW. Correlation of anthropometric measurements,

8. strength, anterior cruciate ligament size, and intercondylar notch characteristics to sex differences in

9. anteriorcruciate ligament tear rates. Am J Sports Med 2001;29(1): 58-66.

10. Chandrashekar N, Slauterbeck J, Hashemi J. Sexbased differences in the anthropometric characteristics of the

11. anterior cruciate ligament And Its relation to intercondylar notch geometry: a cadaveric study. Am J

12. Sports Med 2005; 33(10):1492-8.

13. Fox MG. MR Imaging of the Meniscus: Review, Current Trends, and Clinical Implications.

14. Radiol Clin N Am 2007;45:1033-53. 
15. Rath E, Richmond JC. The menisci: basic science and advances in treatment. Br J Sports Med 2000;34(4):252-7

16. Fithian DC, Kelly MA, Mow VC. Material properties and structurefunction relationships in themenisci. Clin Orthop Relat

17. Res Mar 1990;(252):19-31.

18. Harper KW, Helms CA, Lambert HS, Higgins LD. Radial meniscal tears: significance, incidence, and MR appearance.

19. AJR Am J Roentgenol 2005; 185(6):1429-34.

20. Rohren EM, Kosarek FJ, Helms CA. Discoid lateral meniscus and the frequency of meniscal tears.

21. Skeletal Radiol 2001;30:316-20.

22. Samoto N, Kozuma M, Tokuhisa T, Kobayashi K. Diagnosis of the "large medial meniscus" of the knee on MR imaging.

23. Magn Reson Imaging2006;24:1157-65.

24. Rohren EM, Kosarek FJ, Helms CA. Discoid lateral meniscus and the frequency of meniscal tears. Skeletal Radiol 2001

25. ;30:316-20.

26. Kelly BT, Green DW. Discoid lateral meniscus in children. Curr Opin Pediatr 2002;14:54-61.

27. Helms CA, Major NM, Anderson MW, Kaplan PA, Helms RD. Musculoskeletal MRI. 2nd ed. Philadelphia: Saunders; 2008.

28. Berquist TH. MRI of Musculoskeletal System. 5th ed. Philadelphia: Lippincott William \& Wilkins; 2006.

29. De Smet AA, Norris MA, Yandow DR, Quintana FA, Graf BK, Keene JS. MR diagnosis of meniscal tears of

30. the knee: importance of high signal in the meniscus that extends to the surface. AJR 1993;161:101-7.

31. Rubin DA, Paletta GA. Current concepts and con troversies in meniscal imaging. Magn Reson Imaging

32. Clin N Am 2000;8:243-70.

\section{References}

[1]. The present study attempts to determine the role of magnetic resonance imaging in the evaluation meniscal tears. Knee joint being the largest and most complex weight bearing joint of the body is subject to damage because of its inherent structural complexity and the various types forces it is subjected to. In the setting of traumatic knee joint, MR imaging is the best noninvasive modality for the diagnosis of meniscal tears. Familiarity with the normal anatomy and common pit falls reduces errant interpretation but does not eliminate them entirely. MR imaging of

[2]. knee is considered efficacious especially in the setting of indeterminate clinical findings and can stratify patients, guiding further surgical management. In the present study, 43 cases with history of trauma to knee joint were studied.

[3]. The most common age group to be involved between 15-30 years. The following pattern of meniscal tears were seen Among the meniscal injuries medial meniscal tears were more common and were seen in 20 patients $(46.5 \%)$ than lateral meniscus. Grade III tears were common in both.

[4]. Lee K, Siegel MJ, Lau DM, Hildebolt CF, Matava MJ. Anterior cruciate ligament tear: MR imaging based diagnosis in a paediatric population. Radiology 1999; 213:697-704

[5]. Helms CA, Major NM, Anderson MW, Kaplan PA, Helms RD. Musculoskeletal MRI. 2nd ed. Philadelphia: Saunders; 2008

[6]. Boyd KT, Myers PT. Meniscus preservation: rationale, repair techniques and results. Knee 2003;10:1-11.

[7]. Messner K, Gao J. The menisci of the knee joint: anatomical and functional characteristics, and a rationale for clinicaltreatment. J Anat 1998;193:161-78.Harper KW, Helms CA, Lambert HS, Laurence D. Higgins. Radial Meniscal Tears: Significance, Incidence andMR Appearance. AJR 2005;185:1429-34.

[8]. Brody JM, Lin HM, Hulstyn MJ, Tung GA. Lateral meniscus root tear and meniscus extrusion with anterior cruciateligament tear. Radiology 2006; 239(3):805-10.

[9]. Tuckman GA, Miller WJ, Remo JW, Fritts HM, Rozansky MI. Radial tears of the menisci: MR findings. AJR AmJ Roentgenol 1994;163(2):395-400.

[10]. Rubin DA, Britton CA, Towers JD, Harner CD. Are MR imaging signs of meniscocapsular Separation valid? Radiology 1996;201(3):829-36

[11]. De Maeseneer M, Lenchik L, Starok M, Pedowitz R, Trudell D, Resnick D. Normal and abnormal medial meniscocapsular structures: MR Imaging and sonography in cadavers. AJR Am J Roentgenol 1998;171(4):969-76.

[12]. De Maeseneer M, Shahabpour M, Vanderdood K, Von Roy F, Osteaux M. Medial meniscocapsular separation: MR imaging criteria and Diagnostic pitfalls. Eur J Radiol 2002;41(3):242-53.

[13]. Ruff C, Weingardt J, Russ P, Kilcoyne R. MR imaging patterns of displaced meniscus injuries of the knee AJR 1998;170:63-67.

[14]. Berquist TH. MRI of Musculoskeletal System. 5th ed. Philadelphia: Lippincott William \& Wilkins; 2006.

[15]. Bergin D, Hochberg H, Zoga AC, Qazi N, Parker L, Morrison WB. Indirect Soft-Tissue and Osseous Signs on Knee MRI of Surgically Proven Meniscal Tears AJR 2008; 191:86-92.

[16]. Justice WW, Quinn SF. Error patterns in the MRI evaluation of menisci ofthe knee. Radiology 1995;196:617 -21.

[17]. De Smet AA, Graf BK. Meniscal tears missed on MRI: relationship to meniscal tear patterns and anterior cruciate ligaments tears. AJR 1994;162:905-11.

[18]. Herman LJ, Beltran J. Pitfalls in MRI of the knee. Radiology 1988;167:775-81

[19]. Stroller DW, Martin C, Crues JV, Kaplan L, Mink JH. Meniscal Tears: pathological correlation with MR imaging. Radiology 1987;163:731-5.

[20]. Mesgarzadeh M, Moyer R, Leder DS, Revesz G, Russoniello A, Bonakdarpour A, et al. MR imaging of knee: Expanded classification and pitfalls to the interpretation of meniscal tears. Radiographics 1993;13:489-500.

[21]. Lotysch M, Mink J, Crues JV. Magnetic resonance imaging in the detection of meniscal injuries. Magnetic Resonance Imaging 
1986;4:185.

[22]. Ruff C, Weingardt JP, Russ PD, Kilcoyne RF. MR imaging patterns of displaced meniscus injuries of the knee. AJR Am J Roentgenol 1998; 170(1):63-7.

[23]. Vande Berg BC, Malghem J, Poilvache P, Maldaque B, Lecouvet FE. Meniscal tears with fragments displaced in notch and recesses of knee: MR imaging with arthroscopic comparison. Radiology 2005;234(3): 842-50.

[24]. Magee TH, Hinson GW. MRI of meniscal bucket-handle tears. Skeletal Radiol 1998;27(9):495-9.

[25]. Ververidis AN, Verettas DA, Kazakos KJ, Tilkeridis CE, Chatzipapas CN. Meniscal bucket handle tears: a Retrospective study of arthroscopy and the relation to MRI. Knee Surg Sports Traumatol Arthrosc 2006;14(4):343-9.

[26]. 29. Dorsay TA, Helms CA. Bucket-handle meniscal tears of the knee: sensitivity and specificity of MRI signs.

[27]. Skeletal Radiol 2003;32(5):266- 